Fisioter Bras 2017;18(6):719-26

\title{
ARTIGO ORIGINAL \\ Avaliação do alinhamento postural e extensibilidade muscular pela escala SAROMM em crianças com paralisia cerebral após fisioterapia aquática \\ Evaluation of postural alignment and muscle extensibility with SAROMM scale in cerebral palsy after aquatic physiotherapy
}

Evanir Miranda da Silva*, Tassiane Araújo dos Santos da Silva*, Rodrigo de Souza Balk***, Robson Ricardo Lopes, M.Sc. ${ }^{* *}$, Christian Caldeira Santos ${ }^{* * *}$, Simone Lara**, Susane Graup ${ }^{\star \star \star \star}$

*Acadêmicas de Fisioterapia, Grupo de Pesquisa em Fisioterapia Neurofuncional, Universidade Federal do Pampa, Campus Uruguaiana/ RS, ${ }^{* *}$ Professor Universidade Barão de Mauá, Ribeirão Preto/SP, ${ }^{* * *}$ Professor Assistente, Grupo de Pesquisa em Fisioterapia Neurofuncional, Universidade Federal do Pampa, Campus Uruguaiana/RS, ${ }^{* * * *}$ Professor Adjunto, Grupo de Pesquisa em Fisioterapia Neurofuncional, Universidade Federal do Pampa, Campus Uruguaiana, $R S$

Recebido em 2 de julho de 2017; aceito em 22 de agosto de 2017.

Endereço para correspondência: Rodrigo de Souza Balk, Grupo de Pesquisa em Fisioterapia Neurofuncional, BR 472, Km 592, 97500-970 Uruguaiana RS, Email: rodrigo.balk@gmail.com; Evanir Miranda da Silva: evanir.miranda@yahoo.com.br; Tassiane Araujo dos Santos da Silva: tassi-araujo@hotmail.com; Robson Ricardo Lopes: robsonrlopes@hotmail.com; Christian Caldeira Santos: christiancaldeirasantos@gmail.com; Simone Lara: slarafisio@yahoo.com.br; Susane Graup : susigraup@gmail.com

\section{Resumo}

A paralisia cerebral (PC) é a causa mais prevalente de desordens motoras em crianças no período de maturação estrutural e funcional do sistema nervoso central. A fisioterapia aquática é uma alternativa de tratamento que possibilita a aquisição de funcionalidade na PC de acordo com as capacidades físicas e cognitivas da criança. O estudo teve como objetivo analisar os efeitos da fisioterapia aquática sobre o alinhamento postural e extensibilidade muscular em crianças com diagnóstico de PC. Participaram do estudo quatro crianças com diagnóstico de PC (duas espásticas e duas atetóicas). Foi realizado um protocolo com 10 sessões de Fisioterapia Aquática, incluindo exercícios de fortalecimento de tronco e de membros inferiores (MMII) e de flexibilidade articular, e as crianças foram avaliadas pré e pós-tratamento utilizando a escala Spinal Alignment and Range of Motion Measure (SAROMM). Os quatro sujeitos apresentaram melhora na extensibilidade de MMII, porém não houve melhora no alinhamento de tronco. A fisioterapia aquática apresentou efeitos benéficos sobre extensibilidade muscular de MMII em crianças com PC.

Palavras-chave: paralisia cerebral, Fisioterapia, criança, postura.

\section{Abstract}

Cerebral palsy (CP) is the most prevalent cause of motor disorders in children in the structural and functional maturation of the central nervous system period. The aquatic physical therapy is an alternative treatment which enables the acquisition functionality in the PC according to the physical and cognitive skills of the child. The study aimed to analyze the effects of aquatic physical therapy on postural alignment and muscle extensibility in children with CP diagnosis. The study included four children with diagnosis of CP (two spastic and two athetosis). It carried out a protocol with 10 sessions of Aquatic Physical Therapy, including trunk strengthening exercises and lower limbs (LL) and joint flexibility, and the children were evaluated before and after treatment using the Spinal Scale Alignment and Range of Motion Measure (SAROMM). The four subjects showed improved extensibility of the lower limbs, but there was no improvement in the trunk alignment. The aquatic therapy showed beneficial effects on muscle extensibility lower limbs in children with CP.

Key-words: cerebral palsy, Physical Therapy, child, posture. 
O desenvolvimento motor consiste em uma aquisição contínua de habilidades motoras de acordo com a idade cronológica da criança [1]. Nesse sentido, existem características esperadas para cada faixa etária como reflexos e reações primitivas no primeiro ano de vida, tais como alcançar objetos com as mãos, rolar de prono para supino e vice-versa, apoiar-se nos braços e erguer a cabeça, sentar-se e manter a postura, engatinhar, ficar em pé e, por fim, caminhar sem perder o equilíbrio [2,3]. Por outro lado, o desenvolvimento motor atípico apresenta a falta de uma ou mais, ou, ainda, a permanência de alguma destas características, como acontece na Paralisia Cerebral (PC) [3], em que a criança não consegue ou tem dificuldade de controlar e coordenar seus movimentos e posturas, levando a alterações no desenvolvimento motor [4,5].

Na PC há um desajuste no controle postural normal, assim como nas reações posturais, que são a base para a realização de movimentos contra a gravidade [6]. Estes desajustes posturais podem ser causados por disfunções musculoesqueléticas oriundas da PC, ocasionando déficit na mobilidade e o desenvolvimento de contraturas musculares e deformidades na coluna vertebral (hipercifose, retificação da lordose e escoliose) e extremidades $[7,8]$. Tais deformidades associadas ao desalinhamento de tronco prejudicam 0 desenvolvimento da criança, podendo alterar o padrão de marcha [6].

Neste sentido, um tratamento apropriado pode promover o aumento, tanto quanto possível, das habilidades do indivíduo [9]. Desta forma, visando potencializar os resultados terapêuticos e proporcionar ao indivíduo maior facilidade na realização das atividades por meio da redução da gravidade, a fisioterapia aquática torna-se uma alternativa no tratamento das disfunções causadas pela PC. De fato, a água reduz os efeitos da força da gravidade e com a diminuição do estresse gravitacional nos músculos e articulações, ocorre a redução das informações sensoriais provenientes destes receptores articulares pela ação do empuxo [10].

A fisioterapia aquática possibilita a estabilização e desestabilização do paciente em imersão, devido às propriedades hidrodinâmicas da água que atuam nos movimentos, tendo como principal objetivo para o paciente com PC, a aquisição da funcionalidade de acordo com as suas capacidades físicas e cognitivas [11]. A água facilita a realização de exercícios, proporcionando ao sujeito sensações e facilidades que não são conseguidas em ambiente terrestre [12], devido às propriedades físicas como a pressão hidrostática, a força de empuxo, a termocondutividade e a força de resistência $[13,14]$.

Portanto, o exercício executado no meio aquático representa uma ótima opção para reabilitação e treinamento físico, acarretando melhorias, dentre as quais se destacam o aumento na força muscular, na habilidade para desenvolver as atividades cotidianas, no equilíbrio postural, na flexibilidade, na composição corporal e condicionamento cardiorrespiratório [12,15]. Sendo assim, é necessário realizar uma avaliação funcional dos indivíduos para definir a conduta fisioterapêutica adequada e individualizada a partir das características de cada sujeito e da gravidade da lesão, o que direciona o fisioterapeuta e permite a visualização do antes e depois da realização do tratamento.

Considerando os processos de avaliação, destacamos a Spinal Alignment and Range of Motion Measure (SAROMM), transcrita e validada para o Brasil em 2014 [16] como ferramenta discriminativa para ser usada em reabilitação. Ademais, ela avalia os desvios posturais e a extensibilidade muscular de regiões que fazem conexão direta com o tronco, como o quadril, o ombro e extremidades, como o tornozelo. Este instrumento indica se a criança apresenta o alinhamento e a amplitude de movimento normal ou anormal [17]. Outra ferramenta utilizada nesse contexto, a fim de classificar a gravidade de indivíduos com PC, bem como o nível de habilidade e de limitação funcional dos mesmos é o Gross Motor Function Classification System (GMFCS).

Ao considerar a escassez de estudos sobre os benefícios da fisioterapia aquática em crianças com PC, principalmente, usando métodos de classificação como o GMFCS e avaliação através da SAROMM, este estudo objetiva investigar os efeitos da Fisioterapia Aquática sobre o alinhamento postural e extensibilidade muscular em crianças com diagnóstico de PC, utilizando a escala SAROMM como método de avaliação.

Material e métodos

Este estudo quase-experimental foi realizado em quatro crianças com diagnóstico de PC as quais foram classificadas de acordo com a GMFCS quanto ao nível de habilidades e 
limitações na função motora grossa em crianças e jovens com PC. Este instrumento foi traduzido e adaptado para o Brasil em 2007 [18]. Os critérios de inclusão para participação no estudo foram: diagnóstico de PC, ambos os sexos, faixa etária compreendida entre 02 e 12 anos de idade e ser cognitivamente apto para a realização das tarefas. Os critérios de exclusão foram: cirurgias e problemas ortopédicos recentes, dor na realização das tarefas, alterações de visão ou audição, problemas dermatológicos, lesões na pele e que não completasse 0 protocolo composto por 10 sessões de fisioterapia aquática.

Durante a realização deste estudo as crianças não realizaram nenhum outro tratamento fisioterapêutico concomitante. $O$ instrumento utilizado para avaliação foi uma escala para avaliação do alinhamento postural e extensibilidade muscular denominada SAROMM.

Este trabalho foi submetido ao Comitê de Ética e Pesquisa (CEP) da Universidade local e aprovado sob o parecer número 089461/2015. A participação na pesquisa foi voluntária e um termo de consentimento livre e esclarecido foi assinado pelas (os) responsáveis dos participantes. O quadro I mostra as características dos participantes.

Quadro I - Características descritivas dos sujeitos da amostra do estudo.

\begin{tabular}{|l|l|l|l|l|l|}
\hline Sujeitos & Sexo & Idade & Tipo & Topografia & GMFCS \\
\hline 1 & M & 3 anos & Espástica & Diparesia & Nível IV \\
\hline 2 & M & 3 anos & Espástica & Quadriparesia & Nível V \\
\hline 3 & M & 8 anos & Atetóica & Quadriparesia & Nível V \\
\hline 4 & M & 9 anos & Atetóica & Quadriparesia & Nível V \\
\hline
\end{tabular}

\section{Avaliação}

A avaliação da SAROMM foi transcrita para o Brasil em 2014 [16] e indica se a criança apresenta o alinhamento ou a amplitude de movimento normal ou anormal. Os itens que a escala avalia foram desenvolvidos com conhecimento das variações posturais (sentadoengatinhar-marcha), alinhamento e variedade de limitações de movimento de crianças com PC. Ela possui quatro itens para alinhamento da coluna vertebral e onze para amplitude de movimento e extensibilidade muscular que são testados bilateralmente [17]. No presente estudo, optou-se por utilizar os seguintes aspectos: alinhamento das curvaturas da coluna tronco (lordose cervical, cifose torácica, lordose lombar e escoliose (patológica); quadril (flexão e extensão, rotação interna e externa, adução e abdução); joelho (extensão de joelho e extensibilidade de isquiotibiais) e; tornozelo (plantiflexão e dorsiflexão). A pontuação de cada item varia de zero a quatro pontos, no qual, zero ocorre o alinhamento sem limitações e quatro há desvios graves na coluna vertebral ou limitação na amplitude de movimento ou extensibilidade muscular. O total é a soma dos valores obtidos nas articulações de cada membro e a média é o resultado da divisão dessa soma pela quantidade de articulações avaliadas.

Para a aplicação da SAROMM, a criança foi posicionada para avaliação dos itens 1 a 4 (Alinhamento da coluna vertebral) na posição sentada sobre o tablado ou sobre um banco, sendo sempre necessário que este, estivesse em uma postura alinhada e posicionado com os pés ao chão, distância preservada com ângulo poplíteo a $90^{\circ}$ sem flexão maior de $90^{\circ}$ de quadril, respeitando caso a criança apresentasse contratura muscular que o mantivesse acima de $90^{\circ}$ de flexão de quadril. Para avaliação dos itens 5 a 24, avaliação da amplitude de movimento e extensibilidade muscular, a criança era posicionada em decúbito dorsal sobre o tablado.

A avaliação ocorreu apenas uma vez com cada criança, sendo esta filmada para posterior análise. As filmagens das curvaturas da coluna, os movimentos de extensão e flexão de quadril, extensão de joelho, dorsiflexão e flexão plantar foram realizadas com a câmara posicionada em vista lateral, respeitando o lado a ser testado. Para a avaliação da curva lateral (avaliação da escoliose), a câmera foi posicionada posterior ao tronco. Além disso, para a avaliação dos abdutores, adutores e rotadores internos e externos do quadril, as filmagens foram realizadas com a câmera posicionada na extremidade inferior.

A figura 1 demonstra a avaliação do alinhamento de tronco (1A e 1B) e da extensibilidade muscular de membros inferiores (1C e 1D). 

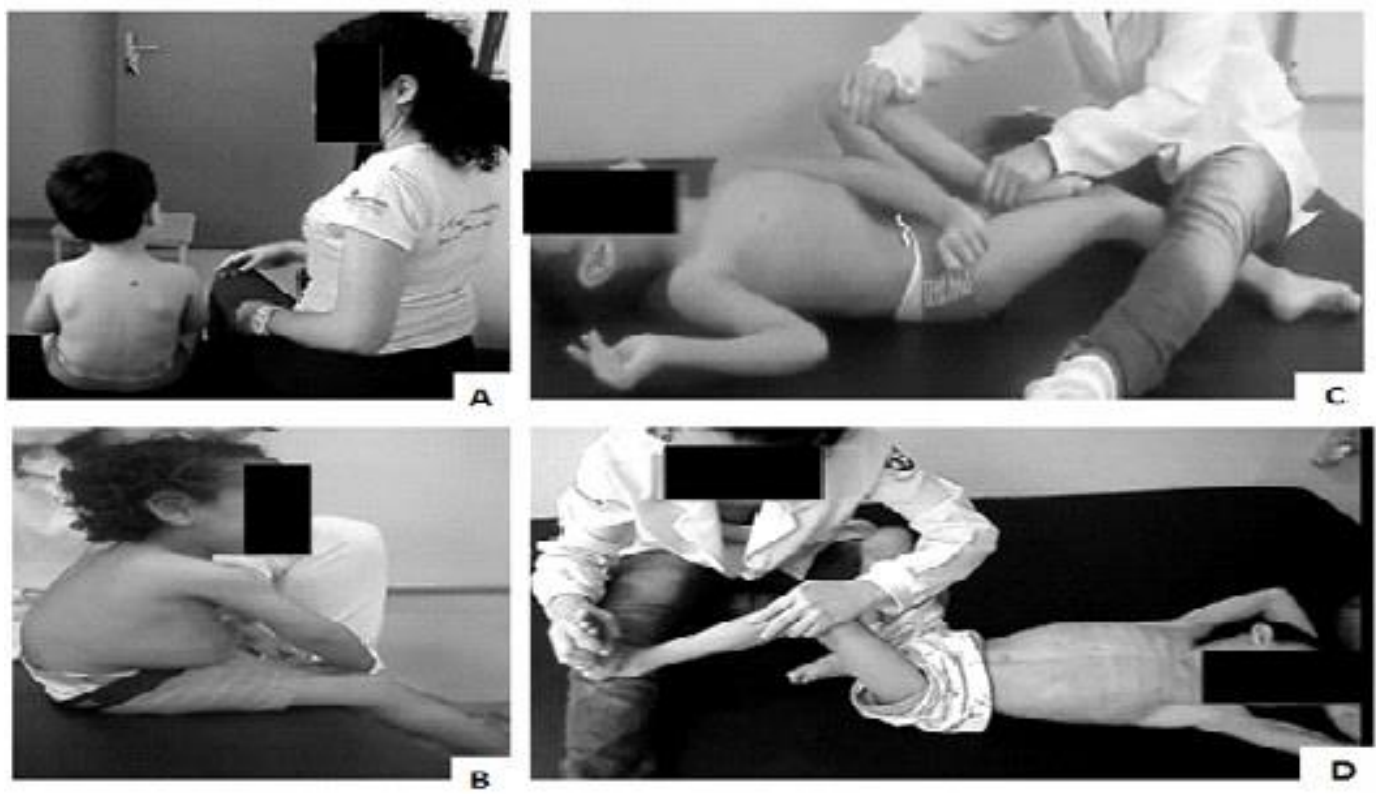

Figura 1 - Avaliação do alinhamento do tronco (1A e 1B) e da extensibilidade muscular (1C e 1D).

\section{Procedimentos}

Foi realizado protocolo terapêutico a partir de estudo realizado por Brancher et al.[10], com ênfase em relaxamento, alongamento e fortalecimento de tronco e membros inferiores e atividades que incentivaram o caminhar, sentar e levantar [11]. O protocolo de exercícios foi elaborado de forma individual, baseado nas limitações e capacidades de cada criança. $O$ tratamento baseou-se em 10 sessões de Fisioterapia Aquática, duas vezes por semana, em uma piscina adaptada com água aquecida a uma temperatura de $32 \pm 1 \stackrel{\circ}{ } \mathrm{C}$, entre outubro e dezembro de 2015. O tratamento foi realizado por 2 pesquisadores previamente treinados. Cada sessão teve duração de uma hora, sendo, 10 minutos para avaliação pré-intervenção, 40 minutos para atendimento, dos quais 5 minutos iniciais e finais eram para adaptação e relaxamento, respectivamente e 10 minutos finais para avaliação pós-intervenção.

\section{Análise estatística}

Os resultados foram analisados através de estatística descritiva utilizando o programa Excel para Windows e comparados intra e interparticipantes, frente aos períodos pré e pósintervenção, que foram descritos e representados por tabelas.

\section{Resultados}

As crianças foram avaliadas pré e pós-tratamento utilizando a SAROMM como método de avaliação, e os resultados obtidos antes e depois foram comparados com dados do próprio sujeito. A análise da Tabela I permite verificar que todas as crianças apresentaram melhora com o tratamento proposto, particularmente, nas articulações de quadril, joelho e tornozelo. Vale destacar que a articulação do quadril era a mais comprometida no pré-teste e, após o tratamento, é possível perceber que, mesmo nos indivíduos com maior nível de déficits, houve uma grande diminuição nos valores totais e médios. Além disso, não foi possível identificar alterações no tronco após a intervenção em nenhum dos sujeitos avaliados. 
Tabela I - Resultados obtidos por meio da SAROOM comparando o antes e depois do tratamento intra sujeitos.

\begin{tabular}{|c|c|c|c|c|c|c|c|c|c|}
\hline & \multicolumn{2}{|c|}{ Sujeito I } & \multicolumn{2}{|c|}{ Sujeito II } & \multicolumn{2}{|c|}{ Sujeito III } & \multicolumn{2}{|c|}{ Sujeito IV } \\
\hline & Articulação & Pré & Pós & Pré & Pós & Pré & Pós & Pré & Pós \\
\hline \multirow[t]{2}{*}{ Tronco } & $\mathrm{T}$ & 0 & 0 & 4 & 4 & 11 & 11 & 4 & 4 \\
\hline & $\bar{x}$ & 0 & 0 & 1 & 1 & 2,75 & 2,75 & 1 & 1 \\
\hline \multirow[t]{2}{*}{ Quadril } & $\mathrm{T}$ & 14 & 2 & 14 & 0 & 22 & 10 & 20 & 8 \\
\hline & $\bar{x}$ & 1,17 & 0,17 & 1,17 & 0 & 1,83 & 1 & 1,67 & 0,83 \\
\hline \multirow[t]{2}{*}{ Joelho } & $\mathrm{T}$ & 8 & 2 & 0 & 0 & 10 & 2 & 12 & 4 \\
\hline & $\bar{X}$ & 2 & 0,5 & 0 & 0 & 2,5 & 0,5 & 3 & 1 \\
\hline \multirow[t]{2}{*}{ Tornozelo } & $\mathrm{T}$ & 6 & 2 & 0 & 0 & 8 & 2 & 4 & 2 \\
\hline & $\bar{x}$ & 1,5 & 0,5 & 0 & 0 & 2 & 0,5 & 1 & 0,5 \\
\hline
\end{tabular}

A tabela II mostra os valores das diferenças entre o pré e o pós-teste, sendo possível identificar que, considerando os valores médios, o joelho foi o que teve maior variação na maioria dos sujeitos, com exceção apenas para o sujeito II que não apresentava limitações nesta articulação.

Tabela II - Valores das diferenças da avaliação na SAROOM entre o pré e o pós-testes de cada sujeito.

\begin{tabular}{|c|c|c|c|c|c|c|c|c|}
\hline \multirow[t]{2}{*}{ Articulação } & \multicolumn{2}{|c|}{ Sujeito I } & \multicolumn{2}{|c|}{ Sujeito II } & \multicolumn{2}{|c|}{ Sujeito III } & \multicolumn{2}{|c|}{ Sujeito IV } \\
\hline & $\Delta \mathrm{T}$ & $\Delta \mathrm{X}$ & $\Delta \mathrm{T}$ & $\Delta \mathrm{X}$ & $\Delta \mathrm{T}$ & $\Delta \mathrm{X}$ & $\Delta \mathrm{T}$ & $\Delta \mathrm{X}$ \\
\hline Tronco & 0 & 0 & 0 & 0 & 0 & 0 & 0 & 0 \\
\hline Quadril & -12 & -1 & -14 & $-1,17$ & -12 & $-0,83$ & -12 & $-0,84$ \\
\hline Joelho & -6 & $-1,5$ & 0 & 0 & -8 & -2 & -8 & -2 \\
\hline Tornozelo & -4 & -1 & 0 & 0 & -6 & $-0,15$ & -2 & $-0,5$ \\
\hline
\end{tabular}

$\Delta \mathrm{T}=$ diferença no total entre o pré e o pós-teste; $\Delta \chi=$ diferença entre as médias do pré e pós- teste.

Discussão

O objetivo deste estudo foi investigar os efeitos da Fisioterapia Aquática sobre 0 alinhamento postural e a extensibilidade muscular em crianças com diagnóstico de PC utilizando a escala SAROMM como método de avaliação. Todos os sujeitos apresentaram melhora nas articulações de quadril, joelho e tornozelo, indicando uma melhor extensibilidade nos grupos musculares que compõem estes componentes articulares.

A água reduz a necessidade de esforço para realizar os movimentos e melhora a qualidade deste durante sua execução $[19,20]$, assim como o exercício no ambiente aquático proporciona melhora na amplitude de movimento e ativação muscular [21]. A melhora na extensibilidade nas articulações de tornozelos apontada pela SAROMM nos 4 sujeitos em nosso estudo pode ter ocorrido, devido ao relaxamento do tônus muscular proporcionado pelas propriedades terapêuticas da água que são benéficas nos casos de espasticidade ou tensão muscular exacerbada [22], pois, a turbulência e temperatura da água interferem no relaxamento muscular e na amplitude de movimento [23], proporcionando melhor extensibilidade à musculatura.

Os sujeitos 2, 3 e 4 não apresentaram melhora no alinhamento de tronco, pois, a classificação no nível IV e V do GMFCS direciona para disfunções mais severas [24] com diminuição da ativação muscular em extensores de tronco, falta de reações de equilíbrio [6] e limitações no controle voluntário dos movimentos e na habilidade de manter posturas antigravitacionais do pescoço e do tronco [25]. Neste sentido, na PC há deficiência no mecanismo de controle postural normal, com alteração nas reações posturais que são base para a realização de movimentos contra a gravidade [26] e as variações clínicas encontradas nas crianças com PC dependem do seu grau de severidade funcional, que está relacionada à persistência dos reflexos primitivos, estiramento muscular e reações posturais que impõem padrões de movimentos rudimentares e controle inadequado da postura [27].

Cabe ressaltar que no presente estudo os sujeitos não obtiveram melhora no alinhamento de tronco após dez sessões de fisioterapia aquática. De fato, é possível inferir que os efeitos de intervenções sobre o tronco necessitem de um maior período de reabilitação [28]. 
Esses autores [28] realizaram 20 sessões de fisioterapia, com base na técnica de reeducação postural global em paciente com PC e perceberam que, após as primeiras 10 sessões, não houve efeitos positivos sobre o tronco e, sim, somente ao final da vigésima sessão de fisioterapia. Em outro estudo, após 30 sessões de fisioterapia aquática em paciente com PC foi possível verificar melhora no equilíbrio devido à perturbação do meio aquático sobre a organização espacial do indivíduo [10].

A reabilitação aquática é proposta para o tratamento de lesões cerebrais e seus problemas associados, como fraquezas musculares, déficit de coordenação motora e equilíbrio $[29,30]$. Considerando este último, é relevante destacar que a manutenção do equilíbrio postural humano depende das informações dos sistemas visuais, proprioceptivos e vestibulares [31].

Assim, Alagesan e Shetty [32] descrevem que intervenções que induzem a um forte estímulo aferente sobre o sistema nervoso, principalmente o proprioceptivo, contribuem para recuperar o atraso motor decorrente da PC, como a hidroterapia, que melhora o equilíbrio postural através da estimulação proprioceptiva [33]. Corroborando, os exercícios aquáticos tornam possível a criação de situações de instabilidade, fornecendo uma grande quantidade de informações sensoriais, que promovem a melhoria nas reações de equilíbrio do corpo [34], uma vez que o alinhamento postural ocorre devido à organização do sistema somatossensorial e vestibular, proporcionando ao indivíduo a autocorreção de algum desajuste que venha a ocorrer [35]. De fato, o aprendizado adquirido dentro da água é transferido para o meio terrestre facilitando a realização dos movimentos e melhor controle do alinhamento postural [13], melhorando a extensibilidade muscular. Desta forma a fisioterapia aquática se torna uma intervenção efetiva em crianças com paralisia cerebral [36] ampliando sua melhora inclusive sobre a saúde mental e atividade cerebral [37].

O presente estudo apresenta algumas limitações. Primeiramente a ausência de um grupo controle e pequena amostra. Em segundo lugar o tempo utilizado no protocolo e ausência de estudos envolvendo a escala SAROMM. Entretanto mesmo com estas limitações é possível verificar resultados positivos na reabilitação seguindo como base para futuros estudos.

Conclusão

A fisioterapia aquática interferiu de forma positiva sobre a reabilitação dos sujeitos da pesquisa, uma vez que melhorou a extensibilidade muscular de membros inferiores em crianças com PC. No entanto, seus efeitos positivos não puderam ser percebidos sobre 0 alinhamento de tronco. Como limitações para este estudo, sentimos falta de estudos mais atuais sobre a aplicabilidade de Fisioterapia Aquática em crianças com PC, visto que a maioria dos trabalhos está relacionada a adultos voltados para outros tipos de patologias neurológicas.

1. Haywood KM, Getchell N. Desenvolvimento motor ao longo da vida. $3^{a}$ ed. Porto Alegre: Artmed; 2004.

2. Rosa Neto F. Manual de avaliação motora. $2^{\mathrm{a}}$ ed. Rev. e Ampl. Florianópolis: DIOESC; 2014. 140p (17).

3. Castilho-Weinert CD, Forti-Bellani LV. Fisioterapia em neuropediatria. Curitiba: Omnipax; 2011.

4. Winnick JP. Educação física e esportes adaptados. 3. ed. Barueri: Manole; 2004.

5. Pirpiris M, Graham HK. Uptime in children with cerebral palsy. J Pediatrics Orthopedics 2004;24(5):521-8.

6. Cunha AB, Polido GJ, Bella GP, Garbellini D, Fornasari CA. Relação entre alinhamento postural e desempenho motor em crianças com paralisia cerebral. Fisioter Pesqui 2009;16(1):22-7.

7. Brasil. Ministério da Saúde. Secretaria de Atenção à Saúde. Departamento de Ações Programáticas Estratégicas. Diretrizes de atenção à pessoa com paralisia cerebral/Ministério da Saúde, Secretaria de Atenção à Saúde, Departamento de Ações Programáticas Estratégicas. Brasília: Ministério da Saúde; 2013.

8. Olama KA, Kassem HI, Aboelazm SN. Impact of aquatic exercise program on muscle tone in spastic hemiplegic children with cerebral palsy. Clin Med J 2015;1(4):138-144.

9. Campbell. Cirurgia ortopédica de Campbell. 8 ed. São Paulo: Manole; 1996. 
10. Brancher EC, Rodrigues RR, Franco PS, Rocha ES, Machado A, Balk RS, et al. Método Halliwick: uma proposta fisioterapêutica na malformação congênita induzida por isotretinoína. Fisioter Bras 2014;15(4):283-8.

11. Silva JB, Branco FR. Fisioterapia aquática funcional. São Paulo: Artes Médicas; 2011.

12. Parreira $P$, Baratella TV, Cohen M, Eds.. Fisioterapia aquática. Barueri: Manole; 2011.

13. Dong KN, Jae-Young L, Hyung-lk S, Nam-Jong P. The effect of aquatic therapy on postural balance and muscle strength in stroke survivors a randomized controlled pilot trial. Clin Rehabil 2008;22:966-76.

14. Freitas Júnior, GC. A Cura pela água - hidrocinesioterapia. Rio de Janeiro: Rio; 2005.

15. Ruoti RG, Morris DM, Cole AJ. Reabilitação Aquática. São Paulo: Manole; 2000.

16. Lopes RR, Pfeifer LI. Adaptação transcultural e estudo preliminar da escala Spinal Alignment and Range of Motion Measure-SAROMM [Dissertação]. Ribeirão Preto: Faculdade de Medicina de Ribeirão Preto; 2014.

17. Bartlett $D$, Purdie B. Testing of the Spinal Alignment and Range of Motion Measure: a discriminative measure of posture and flexibility for children with cerebral palsy. Develop Med Child Neurol 2005;47(11):739-43.

18. Silva DBR, Pfeifer LI, Funayama CAR. GMFCS - E \& R @ Versão Brasileira. Programa de Pós- Graduação em Neurociências e Ciências do Comportamento - Faculdade de Medicina de Ribeirão Preto, Universidade de São Paulo, 2007. [citado 2017 Mai 12]. Disponível em URL: https://canchild.ca/system/tenon/assets/attachments/000/000/075/original/GMFCSER_Translation-Portuguese2.pdf].

19. Garcia MK, Joares EC, Silva MA, Bissolotti RR, Oliveira S, Battistella LR. Conceito Halliwick inclusão e participação através das atividades aquáticas funcionais. Acta Fisiatr 2012;19(3):142-50.

20. Maniu DA, Maniu EA, Benga I. Effects of an aquatic therapy program on vital capacity, quality of life and physical activity index in children with cerebral palsy. HVM Bioflux 2013;5(3):117-24.

21. Orsini M, Feitas MRG, Mello MP, Antonioli RS, Kale N, Eigenheer JF, et al. Hidroterapia no gerenciamento da espasticidade nas paraparesias espásticas de várias etiologias. Rev Neurocienc 2010;18(1):81-6.

22. Carregaro RL, Toledo AM. Efeitos fisiológicos e evidências científicas da eficácia da fisioterapia aquática. Revista Movimenta 2008;1(1):23-7.

23. Oliveira LMM, Braga DM, Oliveira LC, Alves TL, Cyrillo FN, Kanashiro MS. Interferência da fisioterapia aquática no equilíbrio de crianças com paralisia cerebral. Rev Pesq Fisioter 2015;5(2):70-82.

24. Dias ACB, Freitas JC, Formiga CKMR, Viana FP. Desempenho funcional de crianças com paralisia cerebral participantes de tratamento multidisciplinar. Fisiotr Pesqui 2010;17(3):225-9.

25. Brianeze ACGS, Cunha AB, Peviani SM, Miranda VCR, Tognetti VBL, Rocha NACF, et al. Efeito de um programa de fisioterapia funcional em crianças com paralisia cerebral associado a orientações aos cuidadores: estudo preliminar. Fisioter Pesqui 2009;16(1):40-5.

26. Trindade KGR, Celestino ML, Barela AMF. Utilização da informação visual no controle postural de crianças com paralisia cerebral. Fisioter Mov 2013;26(1):107-14.

27. Santos LPD, Golin MO. Evolução motora de crianças com paralisia cerebral diparesia espástica. Rev Neurocienc 2013;21(2):184-92.

28. Silva FP, Martinello M, Medeiros DL, Schwertner DS, Ries LGK. Reeducação postural global em um adulto com paralisia cerebral: um estudo de caso. Fisioter Pesqui 2015;22(1):90-6.

29. Getz M, Hutzler Y, Vermeer A. Effects of aquatic interventions in children with neuromotor impairments: a systematic review of the literature. Clin Rehabil 2006;20(11):927-36.

30. Navarro FM, Machado BBX, Néri AD, Ornellas E, Mazetto AA. A importância da hidrocinesioterapia na paralisia cerebral: relato de caso. Neurocienc 2009;17(4):371-5.

31. D'Hondt E, Deforche B, Bourdeaudhuij I, Gentier I, Tanghe A, Shultz S, et al. Postural balance under normal and altered sensory conditions in normal-weight and overweight children. Clin Biomech (Bristol, Avon) 2011;26(1):84-9.

32. Alagesan J, Shetty A. Effect of modified suit therapy in spastic diplegic cerebral palsysingle blinded randomized controlled trial. Online J Health Allied Scs 2011;9(4):14. 
33. Biasoli MC, Machado CMC. Hidroterapia: aplicabilidades clínicas. Rev Bras Med 2006;63(5):225-37.

34. Avelar NCP, Bastone AC, Alcântara MA, Gomes WF. Effectiveness of aquatic and nonaquatic lower limb muscle endurance training in the static and dynamicbalance of elderly people. Rev Bras Fisioter 2010;14(3):229-36.

35. Passetto MF, Rocha JR, Santana RF, Toledo CAB. Bases neurais do controle postural e o envolvimento da subunidade glur1 nas vias vestibulares em aves. Sci Health 2012;3(3):139-51.

36. Lai CJ, Liu WY, Yang TF, Chen CL, Wu CY, Chan RC. Pediatric aquatic therapy on motor function and enjoyment in children diagnosed with cerebral palsy of various motor severities. J Child Neurol 2015;30(2):200-8.

37. Ryu K1, Ali A2, Kwon M1, Lee C1, Kim Y1, Lee G1, Kim J. Effects of assisted aquatic movement and horseback riding therapies on emotion and brain activation in patients with cerebral palsy. J Phys Ther Sci 2016 ;28(12):3283-7. 